OPEN ACCESS

Edited by:

Chao Tian,

University of Science and Technology of China, China

Reviewed by:

Clifford Cho,

University of Michigan, United States Emma Jane Harris, Institute of Cancer Research (ICR), United Kingdom

${ }^{*}$ Correspondence:

Hong Chen

hongchen@wustl.edu

Specialty section:

This article was submitted to Medical Physics and Imaging,

a section of the journal

Frontiers in Physics

Received: 02 December 2020 Accepted: 09 February 2021

Published: 24 March 2021

Citation:

Yuan J, Ye D, Chen S and Chen H (2021) Therapeutic UltrasoundEnhanced Immune Checkpoint Inhibitor Therapy.

Front. Phys. 9:636985. doi: 10.3389/fphy.2021.636985

\section{Therapeutic Ultrasound-Enhanced Immune Checkpoint Inhibitor Therapy}

\author{
Jinyun Yuan ${ }^{1}$, Dezhuang $\mathrm{Ye}^{1}$, Si Chen ${ }^{1}$ and Hong Chen ${ }^{1,2 *}$ \\ ${ }^{1}$ Department of Biomedical Engineering, Washington University in St. Louis, Saint Louis, MO, United States, ${ }^{2}$ Department of \\ Radiation Oncology, Washington University School of Medicine, Saint Louis, MO, United States
}

Immune checkpoint inhibitors (ICls) are designed to reinvigorate antitumor immune responses by interrupting inhibitory signaling pathways and promote the immunemediated elimination of malignant cells. Although $\mathrm{ICl}$ therapy has transformed the landscape of cancer treatment, only a subset of patients achieve a complete response. Focused ultrasound (FUS) is a noninvasive, nonionizing, deep penetrating focal therapy that has great potential to improve the efficacy of ICls in solid tumors. Five FUS modalities have been incorporated with ICls to explore their antitumor effects in preclinical studies, namely, high-intensity focused ultrasound (HIFU) thermal ablation, HIFU hyperthermia, HIFU mechanical ablation, ultrasound-targeted microbubble destruction (UTMD), and sonodynamic therapy (SDT). The enhancement of the antitumor immune responses by these FUS modalities demonstrates the great promise of FUS as a transformative cancer treatment modality to improve $\mathrm{ICl}$ therapy. Here, this review summarizes these emerging applications of FUS modalities in combination with ICls. It discusses each FUS modality, the experimental protocol for each combination strategy, the induced immune effects, and therapeutic outcomes.

Keywords: focused ultrasound, immune checkpoint inhibitor, antitumor immune response, combination therapy, high-intensity focused ultrasound, low-intensity focused ultrasound

\section{INTRODUCTION}

Immune checkpoint inhibitor (ICI) therapy has revolutionized the paradigm of cancer immunotherapy. Under normal physiological conditions, immune checkpoints are crucial to maintaining immune tolerance. However, in the tumor environment, tumor cells hijack these inhibitory mechanisms to avoid antitumor immune responses. ICIs are monoclonal antibodies that disrupt the engagement of immune checkpoints, which enables tumor-reactive $\mathrm{T}$ cells to overcome inhibitory mechanisms and mount effective antitumor immune responses [1]. The United States Food and Drug Administration (FDA) has approved ICIs that target cytotoxic T lymphocyteassociated protein-4 (CTLA-4), programmed cell death-1 (PD-1), and programmed cell death-ligand 1 (PD-L1) for the treatment of a wide variety of cancers [2]. Despite the clinical success of ICIs, advancing clinical applications of ICIs face challenges related to both efficacy and safety. Most cancer patients are unable to derive durable remission, while $>50 \%$ of cancer patients develop immune adverse events after they receive ICIs [3]. The combination of multiple ICIs with other cancer therapies has improved cancer treatment by enhancing direct tumor killing and indirect antitumor immunity [4].

The past two decades have witnessed exciting breakthroughs in the clinical translations of focused ultrasound (FUS) modalities for cancer treatment [5]. FUS concentrates extracorporeally generated ultrasound energy through the body to a tight focus with an exceptional spatial resolution (on the 
millimeter scale) and deep penetration depth. The focal point can be mechanically and electronically steered in three-dimensional space to form a sonication volume that conforms to the shape of the target. FUS therapy is often performed under the guidance of magnetic resonance imaging or ultrasound imaging [6]. As a promising therapeutic technology, FUS has the unique combined advantages of being noninvasive, nonionizing, nonpharmaceutical, spatially targeted, and deeply penetrating the body. Since 2017, five FUS modalities, including highintensity focused ultrasound (HIFU) thermal ablation [7-11], HIFU hyperthermia [12], HIFU mechanical ablation [13-17], ultrasound-targeted microbubble destruction (UTMD) [18-20], and sonodynamic therapy (SDT) [21, 22], have been investigated in combination with ICIs for treating solid tumors in mouse models. The enhancement of antitumor immune responses by these FUS modalities demonstrated the great promise of FUS as a transformative cancer treatment modality to improve ICI therapy.

In this review, we provide a brief introduction of ICI therapy basics and discuss the challenges facing ICI therapy. We then introduce each FUS-enhanced ICI therapy and summarize the therapeutic outcomes achieved by the combination therapy. Finally, we discuss the limitations of existing studies and provide future perspectives.

\section{Immune Checkpoint Inhibitor Therapy Basics and Challenges}

ICIs bind to immune checkpoints, including CTLA-4, PD-1, and PD-L1, and "release the brakes" on T cells, resulting in anticancer immune responses. CTLA-4 inhibits T-cell activation by attenuating $\mathrm{T}$-cell receptor signaling through competing with the costimulatory molecule CD28 for binding to B7 ligands on antigen-presenting cells (APCs) [23]. PD-1 regulates T-cell activation through interaction with its ligand PD-L1. The engagement of PD-1 and PD-L1 results in a negative costimulatory signal and leads to T-cell apoptosis, anergy, and exhaustion [24]. Efficient ICI therapy requires reactivation and clonal expansion of antigen-experienced $\mathrm{T}$ cells present in the tumor microenvironment (TME) [25]. Initially, naive tumorspecific CD8 T cells are primed by antigen presentation by APCs (often referred to as immune priming) and activated in the presence of costimulatory pathways and cytokines. Tumorspecific CD8 $\mathrm{T}$ cells subsequently differentiate into effector $\mathrm{T}$ cells, undergo clonal expansion, traffic to the TME, and ultimately kill tumor cells. A subset of effector $\mathrm{T}$ cells can differentiate into memory $\mathrm{T}$ cells under the guidance of CD4 $\mathrm{T}$ cells and dendritic cells (DCs) to develop long-term immunologic memory against the tumor.

The introduction of ICI therapy in the clinic has been considered to be a paramount achievement in cancer treatment in the last decade [26]. Since 2011, the FDA has approved ICIs targeting PD-1 (pembrolizumab, nivolumab, and cemiplimab), PD-L1 (atezolizumab, durvalumab, and avelumab), and CTLA-4 (ipilimumab). They have produced remarkable results regarding tumor control in many malignancies, such as melanoma, metastatic non-small-cell lung cancer (NSCLC), head and neck squamous cancers, urothelial carcinoma, gastric adenocarcinoma, mismatchrepair-deficient solid tumors, and classic Hodgkin lymphoma. Many clinical studies with ICIs are currently underway to test their efficacy in various other diseases.

Despite the clinical success of ICIs, ICI therapy faces challenges related to both efficacy and safety. With regard to ICI efficacy, the majority of patients do not benefit from the treatment, and some responders relapse after a period of response. Ongoing studies indicate that both tumor cellintrinsic and tumor cell-extrinsic factors contribute to the resistance mechanisms [27]. Tumor cell-intrinsic factors include lack of tumor-associated antigens (TAAs), ineffective antigen presentation, activation of oncogenic pathways, and insufficient interferon- $\gamma$ (IFN- $\gamma)$ signaling. Tumor cellextrinsic factors are within the TME and include exhausted CD8 $\mathrm{T}$ cells, regulatory $\mathrm{T}$ cells (Tregs), myeloid-derived suppressor cells (MDSCs), and other immunosuppressive cells and factors. With regard to safety, a significant number of patients on ICIs develop immune-related adverse events affecting almost every organ. Immune-related adverse events occur when ICIs result in an immune-based attack on normal tissue. These events, such as dermatitis, thyroiditis, pneumonitis, colitis, hepatitis, and nephritis, are unpredictable, heterogeneous, and in some instances life-threatening. Management of these adverse events remains a challenge [28]. These challenges call for concepts to maximize the clinical benefits of ICIs in combination with other therapies. An abundance of clinical trials are currently underway in evaluating the combination of ICIs with other immunotherapies, chemotherapy, radiotherapy, or targeted therapies. Strategies that can improve antigen presentation and immune recognition, reinforce the activity and infiltration of CD8 T cells, and reduce immunosuppression can potentially be combined with ICIs to improve the efficacy of ICI therapy [27]. Meanwhile, novel drug delivery strategies that enable the targeted delivery of ICIs within the TME have the potential to reduce the toxicities associated with ICIs [29, 30].

FUS is a promising platform technology to be combined with ICIs to improve its efficacy and safety. Various FUS therapeutic modalities have been developed, and some of them have been used in the clinic for the treatment of various diseases (Table 1). Among them, HIFU thermal ablation has been approved by the FDA for the treatment of prostate cancer, uterine fibroids, bone metastasis, and essential tremor and has been used worldwide for the treatment of various diseases. Although other FUS modalities, including HIFU hyperthermia, HIFU mechanical ablation, UTMD, and SDT, have not been approved for clinical use, clinical studies are currently ongoing, with multiple studies already reported. Advances in the clinical applications of these FUS techniques have encouraged new studies to combine FUS with ICIs, as summarized in Table 2 . The effectiveness of FUSenhanced ICI therapy is often demonstrated by increased tumor infiltrated CD8 T cells, decreased tumor volume, and prolonged survival. The systemic immune response of ICI therapy can also be demonstrated by the presence of the abscopal effect, which occurs when the treatment not only shrinks the targeted tumor but also leads to shrinkage of untreated tumors elsewhere in the 
TABLE 1 | Overview of different FUS modalities.

\begin{tabular}{|c|c|c|c|}
\hline Modalities & Commonly used FUS parameters & Physical mechanism & Clinical applications \\
\hline $\begin{array}{l}\text { HIFU thermal } \\
\text { ablation }\end{array}$ & $\begin{array}{l}\text { Continuous (duty cycle }=100 \% \text { ) HIFU with } \\
\text { spatial peak temporal average intensity (ISPTA) } \\
>1000 \mathrm{~W} / \mathrm{cm}^{2} \text { and relative high pressure (a few } \\
\mathrm{MPa} \text { ) to rapidly heat tumor to }>60^{\circ} \mathrm{C} \text { within a few } \\
\text { seconds }\end{array}$ & $\begin{array}{l}\text { Coagulative thermal necrosis due to tissue } \\
\text { absorption of ultrasound energy }\end{array}$ & $\begin{array}{l}\text { The only modality currently approved for use in } \\
\text { the clinic worldwide for the treatment of a variety } \\
\text { of solid malignant tumors (e.g., tumors in the } \\
\text { pancreas, liver, kidney, bone, prostate, and } \\
\text { breast), benign tumors (e.g., uterine fibroids and } \\
\text { fibroadenomata), and non-tumor diseases (e.g., } \\
\text { essential tremor and Parkinson's disease) [32] }\end{array}$ \\
\hline $\begin{array}{l}\text { HIFU } \\
\text { hyperthemia }\end{array}$ & $\begin{array}{l}\text { HIFU with a high duty cycle (up to } 70 \% \text { ) and } \\
\text { relative high pressure (a few } \mathrm{MPa} \text { ) to heat up } \\
\text { tissue to } 40-45^{\circ} \mathrm{C} \text { for up to } 60 \mathrm{~min}[35]\end{array}$ & $\begin{array}{l}\text { Thermally controlled drug release and increasing } \\
\text { blood flow and oxygen }\end{array}$ & $\begin{array}{l}\text { Mutiple reported clinical studies of HIFU } \\
\text { hyperthermia to enhance the delivery of } \\
\text { thermosensitive liposomal doxorubicine in } \\
\text { patients with liver tumors [35] }\end{array}$ \\
\hline $\begin{array}{l}\text { HIFU } \\
\text { mechanical } \\
\text { ablation }\end{array}$ & $\begin{array}{l}\text { HIFU with } I_{\text {SPTA }}>100 \mathrm{~W} / \mathrm{cm}^{2} \text { and a low duty } \\
\text { cycle }(1-2 \%) \text { and extremely high pressure } \\
\text { (>10 MPa) to induce mechanical tissue damage } \\
\text { while minimizing heating [49] }\end{array}$ & $\begin{array}{l}\text { High-amplitude HIFU pusles are used to induce } \\
\text { bubble cavitation to fractionate tissue by } \\
\text { mechanical effects }\end{array}$ & $\begin{array}{l}\text { No reported clinical results, but clinical trials } \\
\text { ongoing for the treatment of primary and } \\
\text { metastatic liver tumors (ClinicalTrials.gov } \\
\text { Identifier: NCT04572633) }\end{array}$ \\
\hline UTMD & $\begin{array}{l}\text { LIFU with ISPTA }<10 \mathrm{~W} / \mathrm{cm}^{2} \text { and a low duty cycle } \\
\text { (e.g., } 10 \%) \text { and relative low pressure }(<1 \mathrm{MPa}) \\
\text { to induce mechanical effects [42] }\end{array}$ & $\begin{array}{l}\text { Targeted and controlled release of therapeutics, } \\
\text { sonoporation for drug/gene delivery, and } \\
\text { mechanical disruption of the blood vessels and } \\
\text { tumor tissue [50] }\end{array}$ & $\begin{array}{l}\text { One reported clinical study of drug delivery using } \\
\text { UTMD in pancreatic patients [51]. Multiple } \\
\text { reported clinical studies on opening the blood- } \\
\text { brain barrier for brain drug delivery in patients } \\
\text { with glioblastoma [52, 53], Alzheimer's disease } \\
\text { [54], and amyotrophic lateral sclerosis [55] }\end{array}$ \\
\hline SDT & $\begin{array}{l}\text { LIFU with } I_{\text {SPTA }}<10 \mathrm{~W} / \mathrm{cm}^{2} \text { and a duty cycle of } \\
10-100 \% \text { to activate sonosensitizers }[56]\end{array}$ & $\begin{array}{l}\text { Sensitizer-dependent sonochemical or } \\
\text { sonophotochemical reactions in an acoustic field } \\
\text { that lead to cytotoxicity [48] }\end{array}$ & $\begin{array}{l}\text { Several reported clinical studies investigated a } \\
\text { combination of light and ultrasound to stimulate a } \\
\text { sensitizer in patients with a variety of cancer [57] }\end{array}$ \\
\hline
\end{tabular}

HIFU (high-intesity focused ultrasound), LIFU (low-intensity focused ultrasound), UTMD (ultrasound-targeted microbubble destruction, SDT (sonodynamic therapy).

body. In the following, each FUS-enhanced ICI therapy is introduced.

\section{High-Intensity Focused Ultrasound Thermal Ablation-Enhanced Immune Checkpoint Inhibitor Therapy}

HIFU thermal ablation induces thermal coagulation by rapidly (in a few seconds) heating tissue at the focus to $>60^{\circ} \mathrm{C}$, often with high-intensity continuous ultrasound waves. Only tissue within the focal region is selectively ablated, while tissue in the ultrasound beam path is spared from ablation [31]. Compared with other local ablative therapies, such as ablative radiotherapy, radiofrequency ablation, and cryotherapy, HIFU thermal ablation is the only noninvasive and nonionizing ablation technique, allowing the procedure to be performed and repeated without the need for surgical implantation of applicators and concerns about radiation-induced toxicities. HIFU thermal ablation causes very few side effects to normal surrounding tissues, and patient comfort and safety are maximized [32]. HIFU thermal ablation has been widely applied for the treatment of a variety of solid tumors, as well as many other benign diseases in the clinic [32]. HIFU thermal ablation has been reported to increase the release of damage-associated molecular patterns (DAMPs) and TAAs, promote DC maturation, increase tumor-infiltrating lymphocytes, and change circulating immunosuppressive cytokine levels [33], suggesting the potential to improve ICI efficacy for tumors that do not respond well to ICIs.

The first study on therapeutic ultrasound-enhanced ICI therapy was reported in 2017 by Silvestrini et al. [7]. They explored whether HIFU thermal ablation could be effectively incorporated with ICIs to boost antitumor immune responses in murine breast cancer models. Breast cancer is often resistant to most chemotherapies and molecular targeted therapies, including ICI therapies [7]. Half of the reported FUS-enhanced ICI studies summarized in Table 2 used murine breast cancer models. In Silvestrini's study, systemic anti-PD-1 antibody ( $\mathrm{APD}-1)$ and local adjuvant, $\mathrm{CpG}$, were administered prior to HIFU thermal ablation for immunotherapy priming. Only with initial immunotherapy priming, coincident HIFU thermal ablation and immunotherapy suppressed tumor growth in both treated and contralateral nontreated tumors and increased the survival rate [7]. The potential mechanisms for the enhanced antitumor response from this multistep protocol were proposed as follows [8]: immunotherapy priming expanded the number of tumorinfiltrating $\mathrm{CD}^{+} \mathrm{T}$ cells and macrophages. The subsequent HIFU thermal ablation released tumor antigens, inflammatory chemokines and cytokines, increased interferon stimulating genes, and altered the local macrophage phenotype. These effects led to cross-presentation and cross-priming mediated by macrophages and DCs, resulting in an effective abscopal response.

The combination of HIFU thermal ablation with ICIs has also been explored to treat colorectal tumors since some of them are not suitable for ICIs [34]. Without immunotherapy priming, the combination of HIFU thermal ablation with ICIs and local adjuvants was able to produce therapeutic benefits in colorectal tumor-bearing mice [9]. HIFU thermal ablation was followed by direct injection of nanoadjuvants into the ablated site and intravenous injection of anti-CTLA-4 antibody ( $\alpha$ CTLA-4). 
TABLE 2 | Summary of therapeutic ultrasound-enhanced ICI therapies.

\begin{tabular}{|c|c|c|c|c|c|}
\hline FUS modality & References & $\begin{array}{l}\text { Cell/mouse } \\
\text { (tumor model) }\end{array}$ & FUS parameters & $\begin{array}{l}\text { Combined FUS and ICI } \\
\text { protocol }\end{array}$ & $\begin{array}{l}\text { Key results } \\
\text { from combination } \\
\text { therapy }\end{array}$ \\
\hline \multirow[t]{5}{*}{$\begin{array}{l}\text { HIFU thermal } \\
\text { ablation }\end{array}$} & Silvestrini et al. [7] & NDL/FVB/n (orthotopic breast cancer) & $\begin{array}{l}3 \mathrm{MHz}, 3.1 \mathrm{MPa}, \mathrm{T}>65^{\circ} \mathrm{C} \text {, target: } \\
\text { circular pattern within } 1-2 \mathrm{~mm} \text { of the } \\
\text { tumor edge }\end{array}$ & $\begin{array}{l}\text { Adjuvant } \mathrm{CpG} \text { (i.t.) injected to single tumor on } \\
\text { days } 21,24,28,31,38,45 \text {; aPD-1 (i.p.) } \\
\text { injected on days 21, 28, and } 35 ; \text { HIFU } \\
\text { thermal ablation applied to tumor on days } \\
31,38,45\end{array}$ & $\begin{array}{l}\text { Distant tumor leukocytes } \uparrow \text { and } \mathrm{CD}^{+} \uparrow \text {; spleen } \\
\text { IFN- } \gamma^{+} \mathrm{CD}^{+} \uparrow \text {; treated and distant tumor volume } \\
\downarrow \text {; survival } \uparrow\end{array}$ \\
\hline & Chavez et al. [8] & $\begin{array}{l}\text { NDL/FVB/n (orthotopic breast cancer) } \\
\text { PyMT/PyVT (spontaneous breast } \\
\text { cancer) }\end{array}$ & $\begin{array}{l}3 \mathrm{MHz}, \mathrm{T}>60^{\circ} \mathrm{C} \text {, target: } 2-3 \mathrm{~mm} \\
\text { within tumor }\end{array}$ & $\begin{array}{l}\text { Adjuvant } \mathrm{CpG} \text { (i.t.) injected to single tumor on } \\
\text { days } 21,24,28,31 ; \alpha \mathrm{PD}-1 \text { (i.p.) injected on } \\
\text { days } 21,28,35, \mathrm{HIFU} \text { thermal ablation } \\
\text { applied to tumor on day } 31\end{array}$ & $\begin{array}{l}\text { Treated tumor interferon-stimulated gene } \\
\text { expression } \uparrow \text {; treated tumor CD169 DCs } \uparrow \text { and } \\
\text { CD169+MPs } \uparrow \text {; distant tumor DCs } \uparrow \text {; spleen } \\
\text { CD169+MPs } \uparrow\end{array}$ \\
\hline & Han et al. [9] & $\begin{array}{l}\text { CT26/BALB/c (subcutaneous } \\
\text { colorectal cancer) }\end{array}$ & $\begin{array}{l}4 \mathrm{MHz}, 43 \mathrm{~W} \text {, FUS on }=1 \mathrm{~s} \text {, FUS off }= \\
5 \mathrm{~s} \text { for a single point, repeat } 20 \text { rounds } \\
\text { to completely eliminate tumor }\end{array}$ & $\begin{array}{l}\text { For metastatic tumor: HIFU thermal ablation } \\
\text { performed to remove tumor on day } 7 \text {; } \\
\text { adjuvants directly injected into ablated site } \\
\text { afterward; } \alpha \text { CTLA-4 (i.v.) injected on days } 8 \text {, } \\
10,12,14 \\
\text { For recurrent tumor: HIFU thermal ablation } \\
\text { performed to remove tumor on day } 7 \text {, } \\
\text { adjuvants directly injected into ablated site } \\
\text { afterward; tumor rechallenged on day } 47 \text {; } \\
\text { aCTLA-4 (i.v.) injected on days } 48,50,52,54\end{array}$ & $\begin{array}{l}\text { Distant tumor } \mathrm{CD}^{+} / \text {regs } \uparrow \text { and MDSCs } \downarrow \text {; } \\
\text { spleen effector memory } \uparrow \text { cell } \\
\left(\mathrm{CD} 3^{+} \mathrm{CD} 44^{H-i} \mathrm{CD} 62^{\mathrm{LO}}\right) \uparrow \text {; serum IFN- } \gamma \uparrow \text { and TNF- } \\
\alpha \uparrow \text {; distant tumor volume } \downarrow \text {; survival } \uparrow \text {; tumor } \\
\text { rechallenge suppressed }\end{array}$ \\
\hline & $\begin{array}{l}\text { Sheybani } \\
\text { et al. [10] }\end{array}$ & $\begin{array}{l}4 \mathrm{~T} 1 / \mathrm{BALB} / \mathrm{c} \text { (subcutaneous breast } \\
\text { cancer) }\end{array}$ & $\begin{array}{l}3 \mathrm{MHz}, 15 \mathrm{~W} \text {, FUS on }=10 \mathrm{~s} \text { for each } \\
\text { sonication, target: } \sim 10-20 \% \text { of total } \\
\text { tumor volume }\end{array}$ & $\begin{array}{l}\text { Every } 3 \text { days for a total of five doses of } \alpha \text { PD- } 1 \\
\text { (i.p.) injected prior to or with HIFU thermal } \\
\text { ablation with gemcitabine }\end{array}$ & Tumor volume $\downarrow$ \\
\hline & Fite et al. [11] & NDL/FVB/n (orthotopic breast cancer) & $\begin{array}{l}3 \mathrm{MHz}, 3.1 \mathrm{MPa}, \mathrm{T}>65^{\circ} \mathrm{C} \text {, circular } \\
\text { pattern within } 1-2 \mathrm{~mm} \text { of the tumor } \\
\text { edge }\end{array}$ & $\begin{array}{l}\text { Adjuvant } \mathrm{CpG} \text { (i.t.) injected to single tumor on } \\
\text { days 21, 24, 28, 30; aPD-1 (i.p.) injected on } \\
\text { days 21, 28, 34, HIFU thermal ablation } \\
\text { applied to tumor on day } 30\end{array}$ & $\begin{array}{l}\text { Treated tumor IL- } 6 \text { and IL- } \beta \text { mRNA } \uparrow \text {; treated } \\
\text { tumor MDSCs } \uparrow \text {; distant tumor MDSCs } \uparrow\end{array}$ \\
\hline $\begin{array}{l}\text { HIFU } \\
\text { hyperthermia }\end{array}$ & $\begin{array}{l}\text { Kheirolomoom } \\
\text { et al. [12] }\end{array}$ & $\begin{array}{l}\text { NDL/FVB/n (orthotopic breast cancer) } \\
\text { PyMT/PyVT (spontaneous breast } \\
\text { cancer) }\end{array}$ & $\begin{array}{l}1.5 \mathrm{MHz} \text {, PNP } 2.5 \mathrm{MPa} \text {, PRF } 100 \mathrm{~Hz} \text {, } \\
\text { pulse length } 0-7 \mathrm{~ms} \text {, heating at } 42^{\circ} \mathrm{C} \\
\text { for } 5 \text { min before and } 20 \text { min post } \\
\text { liposome injection }\end{array}$ & $\begin{array}{l}\text { Copper-doxorubicin-loaded temperature- } \\
\text { sensitive liposomes (i.v.) injected on day } 31 \text {, } \\
\text { HIFU hyperthermia applied to tumor } \\
\text { afterward; adjuvant CpG (i.t.) injected to } \\
\text { single tumor on days } 21,24,28,35,49 \text {; } \\
\text { aPD-1 (i.p.) injected on days } 21,28,35\end{array}$ & $\begin{array}{l}\text { Treated and distant tumor } \operatorname{CD} 8^{+} \uparrow \text {; treated and } \\
\text { distant tumor volume } \downarrow \text {; survival } \uparrow\end{array}$ \\
\hline
\end{tabular}


TABLE 2 | (Continued) Summary of therapeutic ultrasound-enhanced ICI therapies.

\begin{tabular}{llll}
\hline FUS modality & References & $\begin{array}{l}\text { Cell/mouse } \\
\text { (tumor model) }\end{array}$ & FUS parameters \\
\hline $\begin{array}{l}\text { HIFU } \\
\text { mechanical } \\
\text { ablation }\end{array}$ & Wang et al. [13] & $\begin{array}{l}\text { GL261/C57BL/6 (subcutaneous } \\
\text { glioblastoma) }\end{array}$ & $\begin{array}{l}1.1 \mathrm{MHz} \text {, duty cycle 2\%, treatment } \\
\text { duration } 2 \text { min }\end{array}$ \\
\hline
\end{tabular}

Eranki et al. [14] Neuro2a/C57BL/6 (subcutaneous neuroblastoma)
4TI/BALB/c (subcutaneous breas caner); CT26/BALB/c (subcutaneous colorectal cancer)

Nam et al. [15]

Qu et al. [16]

\section{melanoma)}

Hepa1-6/C57BL/6 (subcutaneous hepatoma)

Singh et al. [17] B16F10/C57/BL-6 (subcutaneous melanoma)

\author{
Li et al. [18]
}

Bulner et al. [19] CT26/BALB/c (subcutaneous colorectal cancer)

llovitsh et al. [20] NDL/FVB/n (orthotopic breast cancer)

\section{Combined FUS and IC}

protocol

Perfluorocarbon-filled microshells directly injected to large tumors $\left(400-700 \mathrm{~mm}^{3}\right)$, HIFU mechanical ablation applied to tumo afterward; $\alpha$ PD-1 (i.p.) injected on days 0,2 , $4,6,8,10^{\text {a }}$

1.5 MHz, PNP $14 \mathrm{MPa}$, PRF $1 \mathrm{~Hz}$ pulse duration $13.33 \mathrm{~ms}, 5 \mathrm{~s} /$ location 3 locations, cover $2 \%$ tumor volume

Histotripsy applied to tumor on day 8 ; aCTLA-4 (i.p.) + aPD-L1 (i.p.) injected on days $9,12,15$

$1.5 \mathrm{MHz}$, electrical power $525 \mathrm{~W}, \mathrm{PRF}$ $1 \mathrm{~Hz}$, duty cycle $1 \%$, pulse length $10 \mathrm{~ms}, 50$ pulses

$1 \mathrm{MHz}$, PNP $30 \mathrm{MPa}$, PRF $100 \mathrm{~Hz}, 50$ For B16GP33 tumor, histotripsy applied to pulses, pulse duration $1-2 \mu \mathrm{s}$; total treatment duration $4-15 \mathrm{~min}$ depending on tumor volume

tumor on day 7; $\alpha \mathrm{CTLA}-4$ (i.p.) injected days $6,9,12$

For Hepa1-6 tumor, histotripsy applied to tumor on day 10; aCTLA-4 (i.p.) injected on days $3,6,9,12$

$1.5 \mathrm{MHz}$, acoustic power $450 \mathrm{~W}$, PRF Histotripsy applied to tumor $\left(330-400 \mathrm{~mm}^{3}\right.$ ); $1 \mathrm{~Hz}$, duty cycle $1 \%$, cover $40-50 \%$ of single dose of $\mathrm{CCD} 40$ (i.t.) injected afterward $\begin{array}{ll}1 \mathrm{~Hz} \text {, duty cycle } 1 \% \text {, cover } 40-50 \% \text { of } & \text { single dose of } \alpha \mathrm{CD} 40 \text { (i.t.) injected afterward } \\ \text { the tumor } & \text { (within } 2 \mathrm{~h} \text { ); } 3 \text { dose of } \alpha \mathrm{CTLA}-4 \text { (i.p.) + aPD- }\end{array}$

$1 \mathrm{MHz}, 2.0 \mathrm{~W} / \mathrm{cm}^{2}$, duty cycle $50 \%$, treatment duration $5 \mathrm{~min}$ L1 (i.p.) injected at 3 days interva Docetaxel and aPD-L1-coloaded microbubbles (i.v.) followed by LIFU applied to tumor on days $8,11,14,17,20$ for subcutaneous tumor and on days $6,9,12$ 15, 18 for orthotopic tumor

$1 \mathrm{MHz}$, PNP $1.65 \mathrm{MPa}$, pulse length $0.1 \mathrm{~ms}$, duty cycle $10 \%$, wait 20 s after every 50 pulses, total treatment duration $2 \mathrm{~min}$

$250 \mathrm{kHz}$, PNP $500 \mathrm{kPa}$, PRF $30 \mathrm{~Hz}$, burst length $4 \mathrm{~ms}$, total treatment duration 3 min
LIFU+microbubbles two repeats with $10 \mathrm{~min}$ interval on day 10-12; aPD-1 (i.p.) injected on days $0,3,6$ after LIFU+microbubbles for acute study and 0, 3, 5, 9, 12 for longitudinal study

aCD326-loaded microbubbles and plFN(i.t.) injected followed by LIFU applied to tumor on day 14; $\alpha$ PD-L1 (i.p.) injected on days 11,16

\section{Key results}

from combination

therapy

Tumor $\mathrm{CD}_{4} 5^{+} \uparrow, \mathrm{CD}^{+} \uparrow, \mathrm{CD} 8^{+} \uparrow$, and IFN- $\gamma \uparrow$ tumor size $\downarrow$; survival $\uparrow$; tumor rechallenge rejected

Tumor $\mathrm{CD} 4^{+} \uparrow, \mathrm{CD} \mathrm{a}^{+} \uparrow$ and $\mathrm{CD} 8 \mathrm{a}^{+} \mathrm{CD} 11 \mathrm{C}^{+} \uparrow$ spleen and TDLN CD8 $\alpha^{+} C D 11 c^{+} \uparrow$; spleen CD11 $b^{\text {low }} \uparrow$; spleen effector memory T cells (CD4 or $\mathrm{CD}^{+}{ }^{+} \mathrm{CD} 44^{+}$hiCD62L ${ }^{+}$low) $\uparrow$; serum IFN- $\gamma \uparrow$, IL$6 \uparrow$ and IL-10ป; complete abscopal response; survival $\uparrow$; transferred T cells suppressed tumor in recipients

Tumor $\mathrm{CD}^{+} \uparrow, \mathrm{CD}^{+} \mathrm{CD} 107 \mathrm{a}^{+} \uparrow, \mathrm{CD} 8^{+} \mathrm{PD}-1^{+} \uparrow$ and MDSCs $\downarrow$; tumor volume $\downarrow$; tumor weight $\downarrow$

Tumor CD8 ${ }^{+} \uparrow$; tumor volume $\downarrow$

Tumor growth $\downarrow$; survival $\uparrow$

Tumor TUNEL $\uparrow, \mathrm{Ki}^{2} 7^{+} \downarrow, \mathrm{CD}^{+} \uparrow, \mathrm{CD} 8^{+} \uparrow$; tumo growth $\downarrow$; body weight loss $\downarrow$; survival $\uparrow$

Tumor necrosis $\uparrow$ and growth $\downarrow$; survival $\uparrow$; one survived mouse suppressed tumor rechallenge

Tumor $\mathrm{CD}^{+} \uparrow$ and F4/80 MPs $\uparrow$; treated and distant tumor growth $\downarrow$; survival $\uparrow$

Continued on following page) 


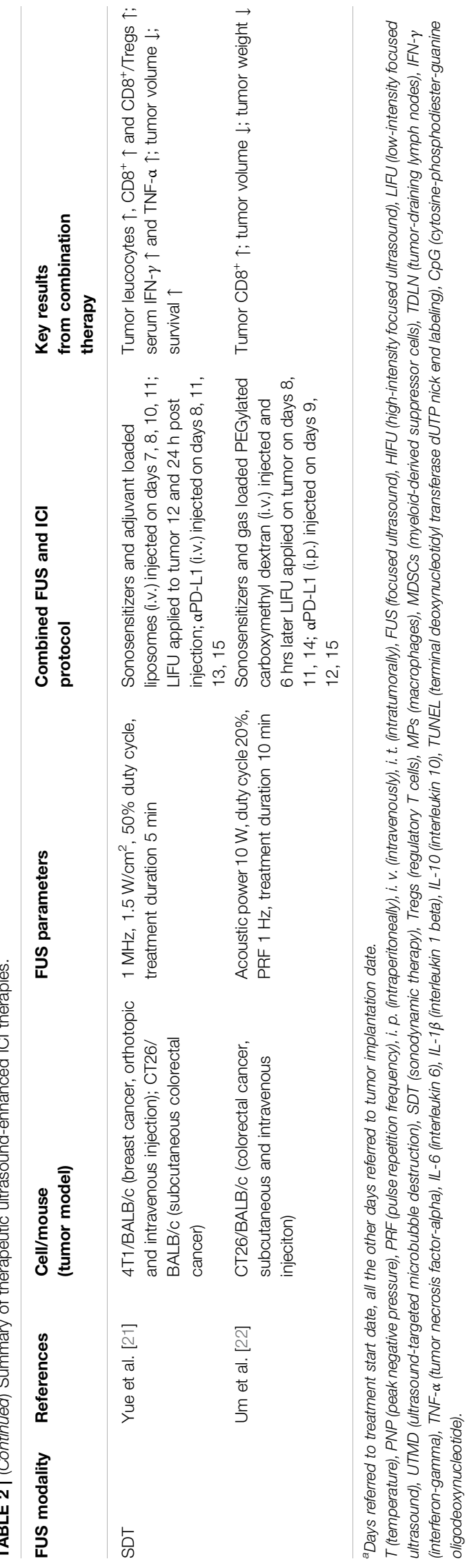

The nanoadjuvants were formed by loading poly (lactic-coglycolic) acid nanoparticles with either a TLR7 agonist (imiquimod, R837) or TLR4 agonist (monophosphoryl lipid A, MPLA). This combination strategy increased the intratumoral CD8 $\mathrm{T}$ cell/Treg ratio, reduced MDSCs within tumors, achieved complete distant tumor eradication, prolonged mouse survival, and prevented tumor recurrence, indicating the generation of sustained immune memory against colorectal tumors. In contrast, none of the mice given HIFU thermal ablation plus either nanoadjuvants or aCLTA-4 survived [9], which suggests that additional agents (e.g., adjuvants, chemotherapeutics) may be required for the success of HIFU thermal ablation and ICI combination treatment. Another study reported that initiating aPD-1 treatment prior to versus shortly after HIFU thermal ablation with chemotherapy did not bear a marked difference in primary tumor growth in a murine breast cancer model [10].

These studies suggest that the optimal protocol for HIFU thermal ablation-enhanced ICI therapy may depend on the tumor model, the type of adjuvants used, with or without immunotherapy priming, and the HIFU ablation protocol. HIFU thermal ablation as a combination therapy with ICIs has the potential limitation that excessive heat generation by HIFU thermal ablation may induce protein denaturation and inactivate antigen presentation [13]. Meanwhile, HIFU thermal ablation was also reported to increase tumor infiltrated MDSCs and Tregs at both directly treated and distant tumors, leading to inhibition of antitumor immunotherapy [11]. These negative effects highlight the complexity of combining HIFU thermal ablation with ICI therapy.

\section{High-Intensity Focused Ultrasound Hyperthermia-Enhanced Immune Checkpoint Inhibitor Therapy}

HIFU hyperthermia raises tissue temperature within the focal region to $40-45^{\circ} \mathrm{C}$ for up to $60 \mathrm{~min}$. It is different from thermal ablation in that hyperthermia is not intended to produce substantial cell death directly. Instead, HIFU hyperthermia is often combined with chemotherapy and radiation therapy or used for local drug release in combination with temperaturesensitive nanoparticles [35]. HIFU hyperthermia can directly promote antigen cross-presentation and tumor-reactive $\mathrm{T}$ cell formation and expansion [36].

Kheirolomoom et al. investigated the combination of HIFU hyperthermia with chemotherapy, $\mathrm{CpG}$, and aPD-1 [12]. HIFU hyperthermia was utilized to control the release of temperaturesensitive liposomes loaded with a chemotherapy drug, doxorubicin (Dox). The liposome carrier was designed to minimize the severe cardiac toxicity of Dox and enhance its delivery efficiency to tumors. Dox released at HIFU hyperthermia-treated tumors enhanced the presentation of tumor-specific antigens at distant tumor sites. Similar to HIFU thermal ablation [7], only with immunotherapy priming by CpG and aPD-1, the combined HIFU hyperthermia, Dox-loaded liposomes, and aPD-1 treatment increased tumor infiltrated CD8 $\mathrm{T}$ cells and achieved complete tumor destruction in both treated and distant tumors as well as prolonged tumor-free 
survival. However, repeated Dox delivery by HIFU hyperthermia either with or without immunotherapy priming reduced the complete response rate, which was considered to be caused by rapid tumor cell death resulting from repeated Dox release that weakened the impact of local antigen and cytokine release. These findings highlighted the importance of the dosing of HIFU hyperthermia-mediated chemotherapy and the timing of immunotherapy to augment ICI efficacy for cancer treatment.

These reported studies [7, 9, 10, 12] suggest that neither HIFU thermal ablation nor HIFU hyperthermia alone is sufficient to enhance ICI efficacy in murine tumor models. Both FUS modalities were found to enhance the release of TAAs and recruitment of CD8 $\mathrm{T}$ cells, but in the absence of additional stimuli (e.g., adjuvants, chemotherapeutics), the recruited CD8 $\mathrm{T}$ cells might not have sufficient antigen cross-presentation and cross-priming mediated by DCs and macrophages [8, 11]. Future studies are needed to investigate the optimal combination therapy by HIFU thermal ablation or hyperthermia with ICIs and adjuvants/chemotherapeutics to achieve systemic, long-term effects for cancer treatment.

\section{High-Intensity Focused Ultrasound Mechanical Ablation-Enhanced Immune Checkpoint Inhibitor Therapy}

HIFU mechanical ablation utilizes short pulse lengths (microsecond to millisecond) and low duty cycles to produce mechanical ablation of tissues while limiting tissue temperature increase. The primary physical mechanism of HIFU mechanical ablation is cavitation, which is defined as the formation, oscillation, and collapse of bubbles in the acoustic field. Cavitation can induce tissue damage by various mechanisms, including microjecting, streaming, and shear stresses [37]. The formation of cavitation in tissue by HIFU can be facilitated by the injection of exogenously made cavitation nuclei, for example, microbubbles or phase-changing materials (e.g., perfluorocarbon). Without the injection of cavitation nuclei, cavitation can be initiated using ultrasound pulses with high tensile pressure, which stretches the tissue and generates cavitation bubbles in situ. When extremely high tensile pressures are generated, HIFU can lead to complete liquefaction of the tumor tissue into submicron fragments, which is named histotripsy [38]. Several reports have shown that HIFU mechanical ablation can cause immunogenic cell death and release tumor debris in situ, promote antigen presentation, and enhance the inflammatory response [33].

The clinical applications of ICIs in brain tumors (e.g., glioblastoma and neuroblastoma) have been challenging, potentially because these tumors harbor a "cold" immune microenvironment that lacks requisite $\mathrm{T}$ cells and sufficient TAAs and contains high densities of immunosuppressive cell populations $[39,40]$. One recent study demonstrated that HIFU mechanical ablation combined with silica microshells mechanically disrupted glioblastoma tumors and augmented the efficacy of aPD-1 [13]. The combination of HIFU mechanical ablation with microshells and $\alpha \mathrm{PD}-1$ increased tumor-infiltrating $\mathrm{CD} 8$ and $\mathrm{IFN}-\gamma^{+} \mathrm{CD} 8 \mathrm{~T}$ cells, prolonged tumor-free survival and protected against tumor rechallenge, suggesting the formation of long-term immune memory against glioblastoma. In a murine neuroblastoma model, Eranki et al. demonstrated that histotripsy potentially transformed immunologically "cold" tumors into responsive "hot' tumors and provided an efficacious adjuvant to ICI therapy [14]. Histotripsy followed by systemic injection of aCTLA-4 and $a \mathrm{PD}-1$ induced significant increases in intratumoral CD4, CD8a, and $\mathrm{CD} 8 \mathrm{a}^{+} \mathrm{DCs}$ in regional lymph nodes and circulating IFN- $\gamma$ and decreases in circulating IL- 10 . Notably, the combination therapy improved long-term survival, achieved complete bilateral tumor regression, and induced an effective long-term immune memory response to suppress subsequent tumor engraftment. Other recent studies found that histotripsy stimulated more potent intratumoral CD8 $\mathrm{T}$ cells and antigen presentation than HIFU thermal ablation in a murine breast cancer model [15] and melanoma model [16]. One recent study showed that combining histotripsy with intratumor anti-CD40 agonist antibody, aCTLA-4, and antiPD-L1 antibody ( $\alpha$ PD-L1) significantly improved the therapeutic efficacy against ICI refractory murine melanoma [17].

These findings [13-16] suggest that HIFU mechanical ablation alone, without the need for adjuvants, is sufficient to enhance ICI therapy for the treatment of cancers that are unresponsive to ICIs. One advantage of HIFU mechanical ablation over HIFU thermal ablation is that tumor fragmentation instead of tumor coagulation may protect TAAs and DAMPs from protein denaturation by excessive heat and stimulate more effective antitumor immune responses [15, 16, 33, 41].

\section{Ultrasound-Targeted Microbubble Destruction-Enhanced Immune Checkpoint Inhibitor Therapy}

There is no consensus regarding the definition of low-intensity focused ultrasound (LIFU). It can be regarded as FUS with pulse intensity similar to that of diagnostic ultrasound. Microbubbles are made of a phospholipid, surfactant, albumin, or synthetic polymer shell filled with a high molecular weight gas with low water solubility. These microbubbles were initially introduced into the clinic as ultrasound contrast agents to enhance ultrasound signals from the blood circulation [42]. Over the past decades, they have been developed into theranostic agents. Their shells can be used for disease-specific targeting and loaded with drugs as carriers for controlled drug release at the LIFU-targeted region. Moreover, microbubble cavitation upon LIFU sonication can generate mechanical forces on surrounding tissue and induce vascular disruption [43].

PD1/PD-L1 ICIs have been used in the clinic for the treatment of NSCLC in combination with chemotherapeutic drugs. However, the combination of these drugs leads to aggravated cardiotoxicity, hematotoxicity, hepatotoxicity, and neurotoxicity [44]. Li et al. used microbubbles as carriers of immunotherapy and chemotherapy drugs to produce antitumor effects while reducing the toxicities of the drug combination [18]. Docetaxel was loaded inside the lipid shell of the microbubbles, and aPD-L1 
was conjugated to the surface of the microbubbles. UTMD improved drug delivery to the tumor potentially through three combined effects: $\alpha \mathrm{PD}-\mathrm{L} 1$ on the surface of the microbubbles specifically targeted the tumor cells; ultrasound sonication ruptured the microbubbles and released the carried drug at the LIFU-targeted tumor site; microbubble cavitation increased tumor permeability and promoted drug penetration across the vessel and into the tumor tissue. As a result, this therapeutic strategy inhibited tumor growth and improved the survival of mice implanted with tumor cells in the lung. It is worth to point out that lung diseases are often considered difficult to treat with FUS because the lungs are air-filled cavities. However, clinical studies have combined ultrasound and microbubbles to enhance drug delivery to the lungs of patients with pneumonia, acute respiratory distress syndrome, and NSCLC [45, 46]. It was proposed that because the diseased areas of the lung are filled with fluid, ultrasound waves could penetrate through the diseased area and leave normal air-filled areas of lung unaffected.

Microbubbles were also used as "anti-vascular" agents to disrupt blood vessels and increase the antitumor effects of ICI therapy of colorectal cancer in a study by Bulner et al. [19]. They found that UTMD alone induced an instant shutdown of blood flow within tumor tissue and resulted in tumor necrosis in a mouse model of colorectal cancer. The combination of UTMD and $\alpha \mathrm{PD}-1$ treatment conferred better tumor growth constriction and a higher survival rate than USMB or aPD-1 alone and rejected subsequent tumor rechallenge. However, the results did not support that the combined UTMD and aPD-1 treatment shifted $\mathrm{T}$-cell subpopulations to a more favorable antitumor state.

In a murine breast cancer model, UTMD produced triple antitumor effects simultaneously: carrying an anti-CD326 antibody to target tumor cells, nonviral gene transduction of IFN- $\beta$ by sonoporation, and tumor debulking by mechanical forces [20]. Such proximity of microbubbles to tumor cells using targeted microbubbles was crucial for effective sonoporation to transfect tumor cells. IFN- $\beta$ expression plus $\alpha \mathrm{PD}-1$ led to a decreased tumor cell population and increased tumorinfiltrating CD8 $\mathrm{T}$ cells. The complete combination treatment attained greater tumor growth reduction in treated and distant tumors and prolonged survival than any partial treatments in the murine breast cancer model.

\section{Sonodynamic Therapy-Enhanced Immune Checkpoint Inhibitor Therapy}

SDT utilizes LIFU to activate sonosensitizers and induces cytotoxicity [47, 48]. Unlike chemotherapy drugs that have massive toxicity on healthy cells, SDT induces tumor cell disruption only at the LIFU-targeted site. Preclinical studies have found that tumor cell debris generated by SDT could provide TAAs for initiating antitumor immunological effects [47, 48]. One report employed SDT using liposomes loaded with sonosensitizers and adjuvants [21]. Strikingly, SDT combined with aPD-L1 eradicated the primary tumor, suppressed distant tumor growth, inhibited whole-body metastasis in murine breast cancer models and produced sufficient immune memory responses to reject subsequent tumor rechallenge in murine breast and colorectal cancer models. The SDT-elicited antitumor effects, immune adjuvantcontaining sonosensitizers, and $\alpha$ PD-L1-mediated systemic antitumor immune response were attributed to the robust antitumor response.

Recently, Um et al. used nanobubbles loaded with a sonosensitizer (chlorin e6) for the treatment of pulmonary metastasis of colorectal cancer [22]. Upon sonication, these nanobubbles caused cell membrane disruption by cavitation, which triggers immunogenic cancer cell death and releases intact DAMPs for in situ cancer vaccination. The combination of aPD-L1 with nanobubbles loaded with the sonosensitizer effectively suppressed primary and metastatic tumors, which suggested that physically induced tumor cell death by the nanobubbles combined with SDT can augment the efficacy of ICIs. More work is needed to determine whether this strategy can improve long-term survival and generate long-lasting immune memory responses against tumor recurrence.

\section{DISCUSSION}

Recent publications have presented exciting and promising results that FUS modalities can improve ICI therapy. Combination therapies were reported to suppress tumor growth, achieve tumor remission, improve long-term survival, and prevent tumor recurrence for cancer types that are not readily responsive to ICI treatments. The field of FUS-enhanced IC therapy is still in its infancy, with all existing studies focused on proofing the concept. Further development of the combination strategy requires a multidisciplinary approach with a proper choice of FUS parameters for particular tumors, a complete examination of the correlation between FUS parameters and antitumor immune effects, a thorough evaluation of the biological mechanisms for therapeutic outcome, and a good understanding of the clinical challenges in cancer immunotherapy.

Although each FUS modality has the capability to improve ICI immunotherapy, it is still unknown which regimen has the greatest potential to combine with ICIs. One major challenge is the inconsistent reporting of FUS parameters and antitumor immune effects, which prevents correlating FUS parameters with antitumor immune effects. It is important to standardize reporting on FUS procedures to include all key parameters, such as ultrasound frequency, intensity, pressure, duty cycle, pulse repetition frequency, sonication target locations, and sonication duration. It is also critical to establish standards in reporting antitumor immune effects to enable comparisons across different studies. Another challenge is that the choice of the optimal FUS modality to improve ICI immunotherapy may depend on tumor type.

The biological mechanisms of each FUS-enhanced ICI therapy remain to be revealed. The reported HIFU thermal ablationenhanced therapy required adjuvants to provide sufficient antigen cross-presentation and cross-priming for CD8 T cells. In contrast, HIFU mechanical ablation alone was sufficient to 
effectively stimulate antitumor immune responses to enhance ICI therapy. There was only one report on HIFU hyperthermiaenhanced ICI therapy. UTMD has great potential to improve ICI therapy through targeted and controlled release of therapeutics, sonoporation, and mechanical disruption of the blood vessels and tumor tissue. SDT induces tumor cell disruption only at the FUStargeted site, resulting in reduced toxicity. Further investigations are warrant to better understand the biological mechanisms of each combination therapy.

FUS-enhanced ICI therapies have already undergone early stage clinical evaluations. Currently, two clinical trials have begun to evaluate the combination of HIFU thermal ablation with pembrolizumab ( $\alpha \mathrm{PD}-1$ ) for the treatment of various advanced solid tumors, such as melanoma, breast cancer, and Merkel cell carcinoma (ClinicalTrials.gov Identifier: NCT04116320 and NCT03237572). The primary outcome will assess a change in the CD8/CD4 T cell ratio in the ablation zone, and the secondary outcome will assess adverse events. Meanwhile, one clinical trial has started evaluating the use of UTMD to enhance the permeability of the blood-brain barrier without causing vascular damage to facilitate the delivery of nivolumab ( $\alpha \mathrm{PD}$ 1) to melanoma metastases in the brain and boost immunity in the brain (NCT04021420).

For the successful clinical translation of FUS-enhanced ICI therapy, we need strong collaboration between ultrasound

\section{REFERENCES}

1. Pardoll DM. The blockade of immune checkpoints in cancer immunotherapy. Nat Rev Cancer (2012) 12:252-64. doi:10.1038/nrc3239

2. Topalian SL, Taube JM, Pardoll DM. Neoadjuvant checkpoint blockade for cancer immunotherapy. Science (2020) 367:eaax0182. doi:10.1126/science. aax0182

3. Sharma P, Allison JP. Dissecting the mechanisms of immune checkpoint therapy. Nat Rev Immunol (2020) 20:75-6. doi:10.1038/s41577-020-0275-8

4. Zappasodi R, Merghoub T, Wolchok JD. Emerging concepts for immune checkpoint blockade-based combination therapies. Cancer Cell (2018) 33: 581-98. doi:10.1016/j.ccell.2018.03.005

5. Tempany CM, McDannold NJ, Hynynen K, Jolesz FA. Focused ultrasound surgery in oncology: Overview and principles. Radiolgy (2011) 259:39-56. doi:10.1148/radiol.11100155

6. Jolesz FA. MRI-guided focused ultrasound surgery. Annu Rev Med.60 (2009). p. 417-30. doi:10.1146/annurev.med.60.041707.170303

7. Silvestrini MT, Ingham ES, Mahakian LM, Kheirolomoom A, Liu Y, Fite BZ, et al. Priming is key to effective incorporation of image-guided thermal ablation into immunotherapy protocols. JCI insight (2017) 2:e90521. doi:10.1172/jci. insight.90521

8. Chavez M, Silvestrini MT, Ingham ES, Fite BZ, Mahakian LM, Tam SM, et al. Distinct immune signatures in directly treated and distant tumors result from TLR adjuvants and focal ablation. Theranostics (2018) 8:3611-28. doi:10.7150/ thno. 25613

9. Han X, Wang R, Xu J, Chen Q, Liang C, Chen J, et al. In situ thermal ablation of tumors in combination with nano-adjuvant and immune checkpoint blockade to inhibit cancer metastasis and recurrence. Biomaterials (2019) 224:119490. doi:10.1016/j.biomaterials.2019.119490

10. Sheybani ND, Witter AR, Thim EA, Yagita H, Bullock TNJ, Price RJ. Combination of thermally ablative focused ultrasound with gemcitabine controls breast cancer via adaptive immunity. J Immunother Cancer (2020) 8:e001008. doi:10.1136/jitc-2020-001008

11. Fite BZ, Wang J, Kare AJ, Ilovitsh A, Chavez M, Ilovitsh T, et al. Immune modulation resulting from MR-guided high intensity focused ultrasound in a engineers and immunologists. Ultrasound engineers can optimize FUS parameters to induce the optimal biological effects that effectively induce antitumor immune responses with minimized side effects. Immunologists can better characterize the resulting antitumor immune responses and therapeutic outcomes. Through appropriate tuning of FUS exposure conditions and comprehensive immunological characterization, the prospect of unmasking the utility of FUS with ICI therapy could be attainable.

\section{AUTHOR CONTRIBUTIONS}

$\mathrm{HC}$ conceived the outline of the review article and edited the manuscript. JY collected the literature, wrote the initial manuscript, and revised the manuscript. DY and SC revised the manuscript. All authors proofread and approved the final manuscript.

\section{FUNDING}

National Institutes of Health (NIH) Grants R01EB027223, R01EB030102, and R01MH116981. The Charlie Teo Foundation and Little Legs Foundation. model of murine breast cancer. Sci Rep (2021) 11:927-15. doi:10.1038/s41598020-80135-1

12. Kheirolomoom A, Silvestrini MT, Ingham ES, Mahakian LM, Tam SM, Tumbale SK, et al. Combining activatable nanodelivery with immunotherapy in a murine breast cancer model. J Control Release (2019) 303:42-54. doi:10.1016/j.jconrel.2019.04.008

13. Wang J, Huang CH, Echeagaray $\mathrm{OH}$, Amirfakhri S, Blair SL, Trogler WC, et al. Microshell enhanced acoustic adjuvants for immunotherapy in glioblastoma. Adv Therap (2019) 2:1900066. doi:10.1002/adtp.201900066

14. Eranki A, Srinivasan P, Ries M, Kim A, Lazarski CA, Rossi CT, et al. Highintensity focused ultrasound (HIFU) triggers immune sensitization of refractory murine neuroblastoma to checkpoint inhibitor therapy. Clin Cancer Res (2020) 26:1152-61. doi:10.1158/1078-0432.CCR-19-1604

15. Nam GH, Pahk KJ, Jeon S, Park HJ, Kim GB, Oh SJ, et al. Investigation of the potential immunological effects of boiling histotripsy for cancer treatment. Adv Therap (2020) 3:1900214. doi:10.1002/adtp.201900214

16. Qu S, Worlikar T, Felsted AE, Ganguly A, Beems MV, Hubbard R, et al. Nonthermal histotripsy tumor ablation promotes abscopal immune responses that enhance cancer immunotherapy. J Immunother Cancer (2020) 8:e000200. doi:10.1136/jitc-2019-000200

17. Singh MP, Sethuraman SN, Miller C, Malayer J, Ranjan A. Boiling histotripsy and in-situ CD40 stimulation improve the checkpoint blockade therapy of poorly immunogenic tumors (2021). p. 11. doi:10.7150/thno.49517

18. Li T, Hu Z, Wang C, Yang J, Zeng C, Fan R, et al. PD-L1-targeted microbubbles loaded with docetaxel produce a synergistic effect for the treatment of lung cancer under ultrasound irradiation. Biomater Sci (2020) 8:1418-30. doi:10. 1039/c9bm01575b

19. Bulner S, Prodeus A, Gariepy J, Hynynen K, Goertz DE. Enhancing checkpoint inhibitor therapy with ultrasound stimulated microbubbles. Ultrasound Med Biol (2019) 45:500-12. doi:10.1016/j.ultrasmedbio.2018.10.002

20. Ilovitsh T, Feng Y, Foiret J, Kheirolomoom A, Zhang H, Ingham ES, et al. Lowfrequency ultrasound-mediated cytokine transfection enhances $\mathrm{T}$ cell recruitment at local and distant tumor sites. Proc Natl Acad Sci USA (2020) 117:12674-85. doi:10.1073/pnas.1914906117

21. Yue W, Chen L, Yu L, Zhou B, Yin H, Ren W, et al. Checkpoint blockade and nanosonosensitizer-augmented noninvasive sonodynamic therapy 
combination reduces tumour growth and metastases in mice. Nat Commun (2019) 10:1-15. doi:10.1038/s41467-019-09760-3

22. Um W, Ko H, You DG, Lim S, Kwak G, Shim MK, et al. Necroptosis-inducible polymeric nanobubbles for enhanced cancer sonoimmunotherapy. Adv Mater (2020) 32:e1907953. doi:10.1002/adma.201907953

23. Rowshanravan B, Halliday N, Sansom DM. CTLA-4: a moving target in immunotherapy. Blood (2018) 131:58-67. doi:10.1182/blood-2017-06-741033

24. Qin W, Hu L, Zhang X, Jiang S, Li J, Zhang Z, et al. The diverse function of PD1/PD-L pathway beyond cancer. Front Immunol (2019) 10:2298-16. doi:10. 3389/fimmu.2019.02298

25. Shi H, Lan J, Yang J. Mechanisms of resistance to checkpoint blockade therapy. Singapore: Springer (2020) doi:10.1007/978-981-15-3266-5_5

26. Robert C. A decade of immune-checkpoint inhibitors in cancer therapy. Nat Commun (2020) 11:10-2. doi:10.1038/s41467-020-17670-y

27. Pitt JM, Vétizou M, Daillère R, Roberti MP, Yamazaki T, Routy B, et al. Resistance mechanisms to immune-checkpoint blockade in cancer: tumorintrinsic and -extrinsic factors. Immunity (2016) 44:1255-69. doi:10.1016/j. immuni.2016.06.001

28. Pauken KE, Dougan M, Rose NR, Lichtman AH, Sharpe AH. Adverse events following cancer immunotherapy: obstacles and opportunities. Trends Immunol (2019) 40:511-23. doi:10.1016/j.it.2019.04.002

29. Riley RS, June CH, Langer R, Mitchell MJ. Delivery technologies for cancer immunotherapy. Nat Rev Drug Discov (2019) 18:175-96. doi:10.1038/s41573018-0006-Z

30. Ye D, Yuan J, Yue Y, Rubin JB, Chen H. Focused ultrasound-enhanced delivery of intranasally administered anti-programmed cell death-ligand 1 antibody to an intracranial murine glioma model. Pharmaceutics (2021). 13(2):1-12. doi:10.3390/pharmaceutics 13020190

31. Kennedy JE. High-intensity focused ultrasound in the treatment of solid tumours. Nat Rev Cancer (2005) 5:321-7. doi:10.1182/blood-2004-10$413510.1038 / \mathrm{nrc1591}$

32. Izadifar Z, Izadifar Z, Chapman D, Babyn P. An introduction to high intensity focused ultrasound: systematic review on principles, devices, and clinical applications. Jcm (2020) 9:460. doi:10.3390/jcm9020460

33. van den Bijgaart RJ, Eikelenboom DC, Hoogenboom M, Fütterer JJ, den Brok MH, Adema GJ. Thermal and mechanical high-intensity focused ultrasound: perspectives on tumor ablation, immune effects and combination strategies. Cancer Immunol Immunother (2017) 66:247-58. doi:10.1007/s00262-016-1891-9

34. Yaghoubi N, Soltani A, Ghazvini K, Hassanian SM, Hashemy SI. PD-1/PD-L1 blockade as a novel treatment for colorectal cancer. Biomed Pharmacother (2019) 110:312-8. doi:10.1016/j.biopha.2018.11.105

35. Zhu L, Altman MB, Laszlo A, Straube W, Zoberi I, Hallahan DE, et al. Ultrasound hyperthermia technology for radiosensitization. Ultrasound Med Biol (2019) 45:1025. doi:10.1016/j.ultrasmedbio.2018.12.007

36. Baronzio GF, Delia Seta R, D’Amico M, Baronzio A, Freitas I, Forzenigo G, et al. Effects of local and whole body hyperthermia on immunity. In: Hyperth cancer treat A prim (2006) p. 247-75. doi:10.1007/978-0-387-33441-7_20

37. Chen H, Kreider W, Brayman AA, Bailey MR, Matula TJ. Blood vessel deformations on microsecond time scales by ultrasonic cavitation. Phys Rev Lett (2011) 106:034301. doi:10.1103/PhysRevLett.106.034301

38. Khokhlova VA, Fowlkes JB, Roberts WW, Schade GR, Xu Z, Khokhlova TD, et al. Histotripsy methods in mechanical disintegration of tissue: towards clinical applications. Int J Hyperthermia (2015) 31:145-62. doi:10.3109/ 02656736.2015.1007538

39. Duan Q, Zhang H, Zheng J, Zhang L. Turning cold into hot: firing up the tumor microenvironment. Trends Cancer (2020) 6:605-18. doi:10.1016/j.trecan.2020.02.022

40. Wang SS, Bandopadhayay P, Jenkins MR. Towards immunotherapy for pediatric brain tumors. Trends Immunol (2019) 40:748-61. doi:10.1016/j.it.2019.05.009

41. Hoogenboom M, Eikelenboom D, den Brok MH, Heerschap A, Fütterer JJ, Adema GJ. Mechanical high-intensity focused ultrasound destruction of soft tissue: working mechanisms and physiologic effects. Ultrasound Med Biol (2015) 41:1500-17. doi:10.1016/j.ultrasmedbio.2015.02.006

42. Chen H, Hwang JH. Ultrasound-targeted microbubble destruction for chemotherapeutic drug delivery to solid tumors. J Ther Ultrasound (2013) 1:10. doi:10.1186/2050-5736-1-10
43. Ferrara K, Pollard R, Borden M. Ultrasound microbubble contrast agents: fundamentals and application to gene and drug delivery. Annu Rev Biomed Eng (2007) 9:415-47. doi:10.1146/annurev.bioeng.8.061505.095852

44. Kanda S, Goto K, Shiraishi H, Kubo E, Tanaka A, Utsumi H, et al. Safety and efficacy of nivolumab and standard chemotherapy drug combination in patients with advanced non-small-cell lung cancer: a four arms phase Ib study. Ann Oncol (2016) 27:2242-50. doi:10.1093/annonc/mdw416

45. Lu B, Sun L, Yan X, Ai Z, Xu J. Intratumoral chemotherapy with paclitaxel liposome combined with systemic chemotherapy: a new method of neoadjuvant chemotherapy for stage III unresectable non-small cell lung cancer. Med Oncol (2015) 32:345-8. doi:10.1007/s12032-014-0345-5

46. Sugiyama MG, Mintsopoulos V, Raheel H, Goldenberg NM, Batt JE, Brochard $\mathrm{L}$, et al. Lung ultrasound and microbubbles enhance aminoglycoside efficacy and delivery to the lung in Escherichia coli-induced pneumonia and acute respiratory distress syndrome. Am J Respir Crit Care Med (2018) 198:404-8. doi:10.1164/rccm.201711-2259LE

47. Wan GY, Liu Y, Chen BW, Liu YY, Wang YS, Zhang N. Recent advances of sonodynamic therapy in cancer treatment. Cancer Biol Med (2016) 13:325-38. doi:10.20892/j.issn.2095-3941.2016.0068

48. McHale AP, Callan JF, Nomikou N, Fowley C, Callan B. Sonodynamic therapy: concept, mechanism and application to cancer treatment. Adv Exp Med Biol (2016) 880:429-50. doi:10.1007/978-3-319-22536-4_22

49. Parsons JE, Cain CA, Abrams GD, Fowlkes JB. Pulsed cavitational ultrasound therapy for controlled tissue homogenization. Ultrasound Med Biol (2006) 32: 115-29. doi:10.1016/j.ultrasmedbio.2005.09.005

50. Roovers S, Segers T, Lajoinie G, Deprez J, Versluis M, De Smedt SC, et al. The role of ultrasound-driven microbubble dynamics in drug delivery: from microbubble fundamentals to clinical translation. Langmuir (2019) 35: 10173-91. doi:10.1021/acs.langmuir.8b03779

51. Dimcevski G, Kotopoulis S, Bjånes T, Hoem D, Schjøtt J, Gjertsen BT, et al. A human clinical trial using ultrasound and microbubbles to enhance gemcitabine treatment of inoperable pancreatic cancer. $J$ Control Release (2016) 243:172-81. doi:10.1016/j.jconrel.2016.10.007

52. Carpentier A, Canney M, Vignot A, Reina V, Beccaria K, Horodyckid C, et al. Clinical trial of blood-brain barrier disruption by pulsed ultrasound. Sci Transl Med (2016) 8:343re2. doi:10.1126/scitranslmed.aaf6086

53. Park SH, Kim MJ, Jung HH, Chang WS, Choi HS, Rachmilevitch I, et al. Safety and feasibility of multiple blood-brain barrier disruptions for the treatment of glioblastoma in patients undergoing standard adjuvant chemotherapy. J Neurosurg (2020) 1-9. doi:10.3171/2019.10.jns192206

54. Lipsman N, Meng Y, Bethune AJ, Huang Y, Lam B, Masellis M, et al. Bloodbrain barrier opening in Alzheimer's disease using MR-guided focused ultrasound. Nat Commun (2018) 9:2336. doi:10.1038/s41467-018-04529-6

55. Abrahao A, Meng Y, Llinas M, Huang Y, Hamani C, Mainprize T, et al. Firstin-human trial of blood-brain barrier opening in amyotrophic lateral sclerosis using MR-guided focused ultrasound. Nat Commun (2019) 10:4373. doi:10. 1038/s41467-019-12426-9

56. Rosenthal I, Sostaric JZ, Riesz P. Sonodynamic therapy-a review of the synergistic effects of drugs and ultrasound. Ultrason Sonochem (2004) 11: 349-63. doi:10.1016/j.ultsonch.2004.03.004

57. Kenyon J, Fulle R, Lewis T. Activated cancer therapy using light and ultrasound - a case series of sonodynamic photodynamic therapy in 115 patients over a 4 Year period. Cdth (2009) 4:179-93. doi:10.2174/157488509789055036

Conflict of Interest: The authors declare that the research was conducted in the absence of any commercial or financial relationships that could be construed as a potential conflict of interest.

Copyright (c) 2021 Yuan, Ye, Chen and Chen. This is an open-access article distributed under the terms of the Creative Commons Attribution License (CC $B Y)$. The use, distribution or reproduction in other forums is permitted, provided the original author(s) and the copyright owner(s) are credited and that the original publication in this journal is cited, in accordance with accepted academic practice. No use, distribution or reproduction is permitted which does not comply with these terms. 